\title{
Pulmonary artery aneurysm and tuberous sclerosis
}

A 52 year old woman with tuberous sclerosis (TS) was referred with an abnormality on the chest radiograph. In her teens she had had a cyst removed from the right upper lobe, the histology of which was consistent with lymphangioleiomyomatosis (LAM). She was a life long nonsmoker.

At presentation she described breathlessness on exertion and intermittent right sided chest pain. Examination revealed cutaneous evidence of TS (adenoma sebaceum, cervical skin tags, and subungal fibromas). A right sided thoracotomy scar was visible; further examination of the chest was normal with no bruits or venous hum. Resting oxygen saturations breathing air were $96 \%$ and pulmonary function tests were within normal limits. A chest radiograph showed a lesion in the right mid zone (fig 1). A CT scan of the thorax revealed the lesion enhanced with contrast (fig 2); also seen were postoperative changes and a few small cysts representative of mild LAM. A magnetic resonance (MR) scan confirmed the presence of a complex circular aneurysm arising from the right pulmonary artery (fig 3). The patient is awaiting embolectomy to repair the aneurysm. She has not been formally screened but no other vascular aneurysms (especially cerebral ones) are apparent.

Pulmonary artery aneurysms (PAAs) are rare; documented aetiologies include infection and structural, cardiac and vascular abnormalities including vasculitis, in particular Bechet's, pulmonary hypertension, and trauma. Presentation is usually with non-specific symptoms although dissection and massive haemoptysis have been reported. PAAs are usually visible on the plain chest radiograph as an enlarging round or fusiform mass, although CT/MR scanning is needed to exclude other pathologies. It is important to identify and treat them as they are unstable and prone to rupture. Treatment options include aneursymectomy, banding or ligation, and non-surgical embolectomy, which is now the treatment of choice.

TS is an autosomal dominant condition with variable penetrance which often arises de novo. It is characterised by hamartomas in multiple organ systems and vascular involve-
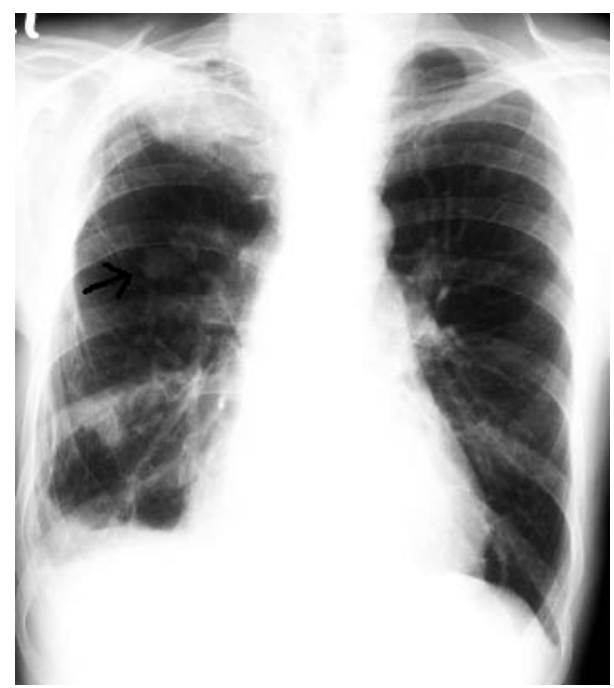

Figure 1 Chest radiograph showing lesion in the right mid zone. ment. Haemangiomas of small blood vessels are relatively common, but aneurysms of medium and large vessels, although rare, have been reported including 10 thoracic and abdominal aortic aneurysms ${ }^{1}$ and a similar number of intracranial aneurysms originating from the internal, anterior, and middle carotid arteries. ${ }^{2}$ Brachial and axillary artery aneurysms are also recognised but are usually asymptomatic. ${ }^{3}$

This case represents the first reported PAA seen in connection with TS and is due to the dysplastic nature of the disease. Although PAAs are rare, it is important to recognise and treat them as their tendency to rupture can be prevented by early intervention.

N J Burrows, S R Johnson

Queens Medical Centre, University Hospital, Nottingham, UK. nicolajb@doctors.net.uk

\section{REFERENCES}

1 Jost CJ, Gloviczki P, Edwards WD, et al. Aortic aneurysms in children and young adults with tuberous sclerosis: report of two cases and review of the literature. J Vasc Surg 2001;33:639-42.

2 Blumenkopf B, Huggins M. Tuberous sclerosis and multiple cranial aneurysms: case report. Neurosurgery 1985;17:797-800.

3 Hite S, Kuo J, Cheung E. Axillary artery aneurysm in tuberous sclerosis: crosssectional image findings. Pediatr Radiol 1998;28:554-6.

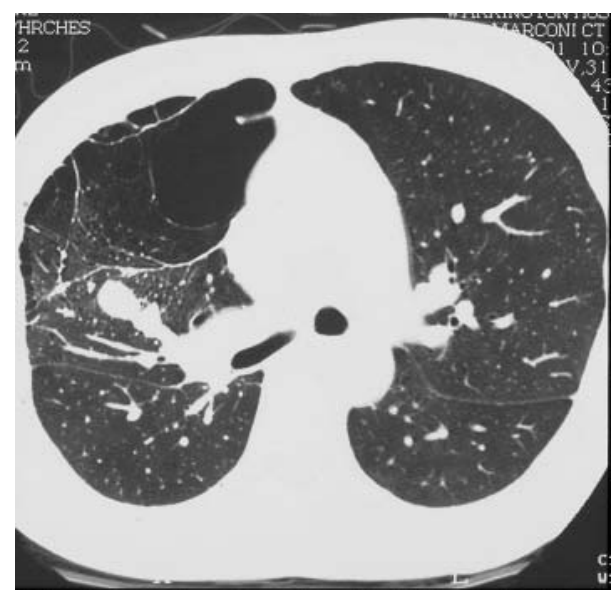

Figure 2 CT scan of thorax showing lesion enhanced with contrast.

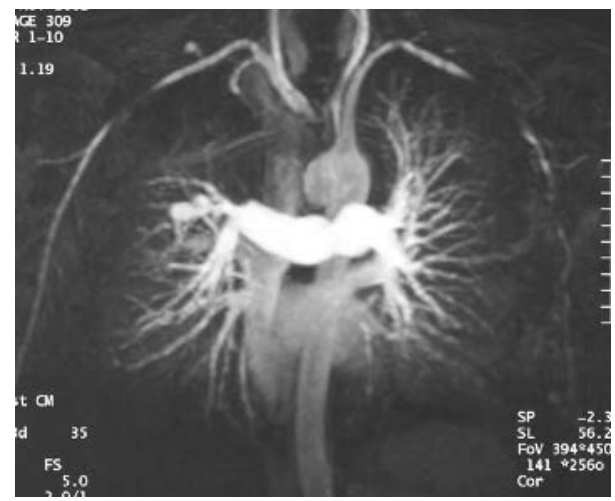

Figure 3 MR scan showing a complex circular aneurysm arising from the right pulmonary artery. 